\title{
GRAPH PRODUCTS OF RIGHT CANCELLATIVE MONOIDS
}

\author{
JOHN FOUNTAIN ${ }^{\bowtie}$ and MARK KAMBITES
}

(Received 2 May 2008; accepted 14 November 2008)

Communicated by M. G. Jackson

\begin{abstract}
Our first main result shows that a graph product of right cancellative monoids is itself right cancellative. If each of the component monoids satisfies the condition that the intersection of two principal left ideals is either principal or empty, then so does the graph product. Our second main result gives a presentation for the inverse hull of such a graph product. We then specialize to the case of the inverse hulls of graph monoids, obtaining what we call 'polygraph monoids'. Among other properties, we observe that polygraph monoids are $F^{*}$-inverse. This follows from a general characterization of those right cancellative monoids with inverse hulls that are $F^{*}$-inverse.
\end{abstract}

2000 Mathematics subject classification: primary 20M10.

Keywords and phrases: graph product, right cancellative monoid, graph monoid, inverse hull, $F *$-inverse monoid.

\section{Introduction}

Graph products of groups were introduced by Green in her thesis [14] and have since been studied by several authors, for example, [15] and [8]. In these two papers, passing reference is made to graph products of monoids, which are defined in the same way as graph products of groups and have been studied specifically by, among others, Veloso da Costa [31, 32] and Fohry and Kuske [13].

In this paper we are interested in graph products of right cancellative monoids. Free products and restricted direct products are special cases of graph products, and a free or (restricted) direct product of right cancellative monoids is again right cancellative. In Section 1, in our first main result, we generalize these observations to obtain a corresponding result for graph products.

We then concentrate on right cancellative monoids in which the intersection of two principal left ideals is either principal or empty. This property holds if and only if any two elements which have a common left multiple have a least common left multiple

(C) 2009 Australian Mathematical Publishing Association Inc. 1446-7887/2009 \$16.00 
(see the end of Section 1), and so, following the terminology from ring theory (see, for example, [1]) we call these monoids left LCM monoids. A useful concept in the study of such monoids is the notion of the inverse hull of a right cancellative monoid. In Section 2, after generalities on inverse hulls, we give several (known) characterizations of inverse hulls of left LCM monoids and use them to show that a graph product of left LCM monoids is itself left LCM. We then consider presentations for inverse hulls of graph products of left LCM monoids. In Section 3 we specialize the presentation to the case where each component monoid is free on one generator, obtaining what we call polygraph monoids, generalizing the polycyclic monoids discussed in [18, Ch. 9].

In the final section, we concentrate on left LCM monoids with two-sided cancellation. Among these monoids we characterize those with an inverse hull that is $F^{*}$-inverse (see Section 4 for the definition), and observe that, in particular, polygraph monoids are $F^{*}$-inverse.

We assume that the reader is familiar with the basic ideas of semigroup theory (see, for example, $[7,16,18])$.

\section{Graph products}

For us, a graph $\Gamma=(V, E)$ is a set $V$ of vertices together with an irreflexive, symmetric relation $E \subseteq V \times V$ whose elements are called edges. In particular, $\Gamma$ is loop free. We say that $u$ and $v$ are adjacent in $\Gamma$ if $(u, v) \in E$. For each $v \in V$, let $M_{v}$ be a monoid; whenever necessary we can, without loss of generality, assume the monoids $M_{v}$ are disjoint. We denote the free product of the $M_{v}$ by $\prod^{\star} M_{v}$ and write $x \cdot y$ for the product of $x, y \in \prod^{\star} M_{v}$.

We define the graph product $\Gamma_{v \in V} M_{v}$ of the $M_{v}$ to be the quotient of $\prod^{\star} M_{v}$ factored by the congruence generated by the relation

$$
R_{\Gamma}=\left\{(m \cdot n, n \cdot m) \mid m \in M_{u}, n \in M_{v} \text { and } u, v \text { are adjacent in } \Gamma\right\} .
$$

Alternatively, if for each $M_{v}$ we have a presentation $\left\langle A_{v} \mid R_{v}\right\rangle$, then $\Gamma_{v \in V} M_{v}$ is the monoid with presentation $\langle A \mid R\rangle$ where

$$
A=\bigcup_{v \in V} A_{v} \quad \text { and } \quad R=\bigcup_{(u, v) \in E}\left\{a b=b a \mid a \in A_{u}, b \in A_{v}\right\} \cup \bigcup_{v \in V} R_{v} .
$$

For the rest of this section we will write $M$ for $\Gamma_{v \in V} M_{v}$. The $M_{v}$ are called the components of $M$, and we denote multiplication in both $M$ and its components by concatenation. It follows from Theorem 1.1 below that the latter embed naturally in the former, and so there should be no cause for confusion.

If the graph has no edges, $M$ is the free product of the $M_{v}$, and at the other extreme, if the graph is complete, $M$ is their restricted direct product.

A special case of interest is when all the $M_{v}$ are isomorphic to the additive monoid of nonnegative integers. The graph product is then called a graph monoid and denoted by $M(\Gamma)$. Graph monoids are also known variously as free partially commutative 
monoids, right-angled Artin monoids, and trace monoids. These monoids and the corresponding groups have been extensively investigated (see, for example, [12] for monoids and [4] for groups).

Now let $X$ be the disjoint union of the $M_{v} \backslash\{1\}$, and for $m \in M_{v} \backslash\{1\}$ write $C(m)=v$. We denote the product in the free monoid $X^{*}$ by $x \circ y$ to distinguish it from the products in $M$ and the $M_{v}$. Clearly there is a canonical surjective homomorphism $\sigma: X^{*} \rightarrow M$ so that each element $a$ of $M$ can be represented by an element of $X^{*}$, called an expression for $a$. If $x_{1} \circ x_{2} \circ \cdots \circ x_{n} \in X^{*}$ is an expression for $a \in M$, the $x_{i}$ are the components of the expression, and if $C\left(x_{i}\right)=v$, then $x_{i}$ is a $v$-component. If $x_{i}$ and $x_{i+1}$ are both $v$-components, then we may obtain a shorter expression for $a$ by, in the terminology of [15], amalgamating $x_{i}$ and $x_{i+1}$ : if $x_{i}, x_{i+1} \in M_{v}$ and $x_{i} x_{i+1}=1$, delete $x_{i} \circ x_{i+1}$; otherwise replace it by the single element $y_{i}$ of $M_{v}$ where $y_{i}=x_{i} x_{i+1}$ in $M_{v}$.

If $\left(C\left(x_{j}\right), C\left(x_{j+1}\right)\right) \in E$ for some $j$, then we may obtain a different expression for $a$ by replacing $x_{j} \circ x_{j+1}$ by $x_{j+1} \circ x_{j}$. Again we follow [15] and call such a move a shuffle. Two expressions are shuffle equivalent if one can be obtained from the other by a sequence of shuffles.

A reduced expression is an element $x_{1} \circ x_{2} \circ \cdots \circ x_{n} \in X^{*}$ which satisfies the following condition: whenever $i<j$ and $C\left(x_{i}\right)=C\left(x_{j}\right)$, there exists $k$ with $i<k<j$ and $\left(C\left(x_{i}\right), C\left(x_{k}\right)\right) \notin E$. Notice that no amalgamation is possible in a reduced expression, and that a shuffle of a reduced expression is again a reduced expression. The following is the monoid version of a result of Green [14] which can also be deduced easily from [31, Theorem 6.1].

THEOREM 1.1. Every element of $M$ is represented by a reduced expression. Two reduced expressions represent the same element of $M$ if and only if they are shuffle equivalent.

The length of an expression is its length as an element of the free monoid $X^{*}$; it is clear that shuffle equivalent expressions have the same length, and so, in view of the theorem, all reduced expressions representing a given element of $M$ have the same length. We shall use this observation without further comment, but we note that it also allows us to define the length of an element of $M$ to be the length of any reduced expression representing it. An easy consequence of the notion of length is the following corollary which we record for later use. First, we recall that a subset $U$ of a monoid $M$ is right unitary in $M$ if, for all elements $m \in M$ and $u \in U$, we have $m \in U$ if $m u \in U$. There is a dual notion of left unitary, and $U$ is unitary in $M$ if it is both right and left unitary.

COROLlaRY 1.2. Each $M_{v}$ is a unitary submonoid of $M$.

PROOF. If $c \in M_{v}, a \in M$ and $a c \in M_{v}$, then $a c$ must have length 1 (or 0 ) and it follows that $a \in M_{v}$. Thus $M_{v}$ is right unitary in $M$ and, similarly, it is left unitary. 
It is natural to ask how properties of $M$ are related to the corresponding properties of the $M_{v}$. Several such questions are considered in [13, 31,32]. Our interest is in right cancellative monoids which do not seem to have been studied in this context. If $M$ is right cancellative, then so too are the $M_{v}$ since they are submonoids of $M$. Our first aim is to show the converse, that is, if all the $M_{v}$ are right cancellative, then so is $M$. Towards this end we introduce the following terminology.

Let $a, a^{\prime} \in M, v \in V$ and $c \in M_{v} \backslash\{1\}$. We say that $a$ has final $v$-component $c$ and final $v$-complement $a^{\prime}$ if $a$ admits a reduced expression $a_{1} \circ a_{2} \circ \cdots \circ a_{m} \circ c$ such that $a_{1} a_{2} \ldots a_{m}=a^{\prime}$. We say that $a$ has final $v$-component 1 and final $v$-complement $a$ if $a$ has a reduced expression $a_{1} \circ \cdots \circ a_{m}$ such that either

(i) $\quad C\left(a_{j}\right) \neq v$ for all $j$, or

(ii) there exists $k$ with $\left(C\left(a_{k}\right), v\right) \notin E$ and $C\left(a_{j}\right) \neq v$ for all $j \geqslant k$.

Of course, we may define the dual notions of initial $v$-component and initial $v$-complement in the obvious way.

Proposition 1.3. For each vertex $v$, each element of $M$ has exactly one final $v$-component and exactly one final v-complement.

PROOF. For existence, suppose that $x \in M$ and let

$$
a_{1} \circ \cdots \circ a_{m}
$$

be a reduced expression for $x$. If condition (i) or (ii) applies, then, by definition, $x$ has final $v$-component 1 and final $v$-complement $x$. Otherwise, there is a largest integer $j$ with $C\left(a_{j}\right)=v$. If $\left(C\left(a_{k}\right), v\right) \notin E$ for some $k>j$, then condition (ii) holds. Hence $\left(C\left(a_{k}\right), v\right) \in E$ for all $k>j$, and it follows easily that one can shuffle $a_{j}$ to the end to obtain a reduced expression

$$
a=a_{1} \circ \cdots \circ a_{j-1} \circ a_{j+1} \circ \cdots \circ a_{m} \circ a_{j}
$$

so that $x$ has final $v$-component $a_{j}$ and final $v$-complement $a_{1} \ldots a_{j-1} a_{j+1} \ldots a_{m}$.

For uniqueness, suppose first, towards a contradiction, that $x$ has distinct final $v$-components 1 and $d \neq 1$. Then $x$ has reduced expressions $a=a_{1} \circ \cdots \circ a_{m}$ and $b=b_{1} \circ \cdots \circ b_{n} \circ d$ where either

(i) $\quad C\left(a_{j}\right) \neq v$ for all $j$, or

(ii) there exists $k$ with $\left(C\left(a_{k}\right), v\right) \notin E$ and $C\left(a_{j}\right) \neq v$ for all $j>k$.

By Theorem 1.1, $b$ can be obtained from $a$ by a sequence of shuffles. But clearly in case (i) such a shuffle can never introduce a $v$-component, while in case (ii) no such shuffle can change the fact that there exists $a_{k}$ with $\left(C\left(a_{k}\right), v\right) \notin E$ and $C\left(a_{j}\right) \neq v$ for all $j>k$. Since $b$ does not satisfy either of the conditions (i) or (ii), this gives a contradiction.

Suppose now that $x$ has reduced expressions

$$
a=a_{1} \circ \cdots \circ a_{m} \circ c
$$


and

$$
b=b_{1} \circ \cdots \circ b_{m} \circ d
$$

where $c, d \in M_{v}, c \neq 1, d \neq 1$. By Theorem $1.1, b$ can be obtained from $a$ by a sequence of shuffles. It is clear that no such shuffle can change the value of the last $v$-component, so we must have $c=d$.

We now turn our attention to showing that final $v$-complements are unique. If the (unique) final $v$-component of $x$ is 1 then, by definition, $x$ is the (unique) final $v$-complement of itself, so there is nothing to prove. So suppose that $x$ has final $v$-component $c \neq 1$, and that there are reduced expressions

$$
a=a_{1} \circ \cdots \circ a_{m} \circ c
$$

and

$$
b=b_{1} \circ \cdots \circ b_{m} \circ c
$$

for $x$. Now by Theorem 1.1, there is a sequence of shuffles which takes $a$ to $b$. Clearly, just by removing those applications which involve the final $v$-component $c$ of the word, we obtain a sequence of shuffles which can be applied to $a_{1} \circ \cdots \circ a_{m}$ to yield $b_{1} \circ \cdots \circ b_{m}$. Since these expressions are reduced, it follows by Theorem 1.1 again that $a_{1} \circ \cdots \circ a_{m}$ and $b_{1} \circ \cdots \circ b_{m}$ represent the same element. Thus, $x$ has exactly one final $v$-complement.

LEMma 1.4. Let $a \in M$ and $c \in M_{v}$. Suppose that a has final $v$-component $d$ and final $v$-complement $a^{\prime}$. Then ac has final $v$-component $d c$ and final $v$-complement $a^{\prime}$.

PROOF. Suppose first that $a$ has final $v$-component $d \neq 1$. Then $a$ has a reduced expression of the form

$$
a_{1} \circ a_{2} \circ \cdots \circ a_{m} \circ d
$$

where $a_{1} \circ \cdots \circ a_{m}$ is a reduced expression for $a^{\prime}$. If $d c \neq 1$ then clearly

$$
a_{1} \circ a_{2} \circ \cdots \circ a_{m} \circ(d c)
$$

is a reduced expression for $a c$, from which the required result is immediate. On the other hand, if $d c=1$ then

$$
a_{1} \circ a_{2} \circ \cdots \circ a_{m}
$$

is a reduced expression for $a c=a^{\prime} d c=a^{\prime}$. It follows easily from the fact that (1) is reduced that either this expression contains no $v$-components, or there exists $k$ such that $\left(C\left(a_{k}\right), v\right) \notin E$ and $a_{j} \notin v$ for all $j \geqslant k$. Thus, $a c$ has final $v$-component 1 and final $v$-complement $a^{\prime}$, as required.

Now consider the case in which $a$ has final $v$-component $d=1$. Then $a$ has a reduced expression

$$
a_{1} \circ a_{2} \circ \cdots \circ a_{m}
$$

where $a=a^{\prime}=a_{1} a_{2} \ldots a_{m}$ and either 
(i) $\quad C\left(a_{j}\right) \neq v$ for all $j$, or

(ii) there exists $k$ with $\left(C\left(a_{k}\right), v\right) \notin E$ and $C\left(a_{j}\right) \neq v$ for all $j \geqslant k$.

In both cases, it is easy to check that $a_{1} \circ a_{2} \circ \cdots \circ a_{m} \circ c$ is a reduced expression for $a c$, from which it follows that $a c$ has final $v$-component $d c=c$ and final $v$-complement $a=a^{\prime}$ as required.

THEOREM 1.5. A graph product of right (left, two-sided) cancellative monoids is right (left, two-sided) cancellative.

PROOF. We prove the result for right cancellative monoids. The corresponding result for left cancellative monoids is proved similarly using initial $v$-components and complements, and the result for cancellative monoids is an immediate consequence of the one-sided results.

First observe that, since the graph product monoid is generated by elements from the embedded components, it suffices to show that elements of the embedded components are right cancellable, that is, that $a c=b c$ implies $a=b$ whenever $c$ belongs $M_{v}$ for some $v \in V$.

Suppose that $a$ and $b$ have (unique) final $v$-components $d$ and $e$ respectively, and (unique) final $v$-complements $a^{\prime}$ and $b^{\prime}$ respectively. Then by the preceding lemma, $a c$ has final $v$-component $d c$ and final $v$-complement $a^{\prime}$, while $b c$ has final $v$-component $e c$ and final $v$-complement $b^{\prime}$.

Since $a c=b c$, we deduce from Proposition 1.3 that $d c=e c$ and $a^{\prime}=b^{\prime}$. But $d, e$ and $c$ lie in $M_{v}$, which by assumption is right cancellative, so we deduce that $d=e$, and hence that $a=a^{\prime} d=b^{\prime} e=b$ as required to complete the proof.

We next consider the question of whether a graph product of monoids each of which is embeddable in a group is itself embeddable in a group. A positive answer is a consequence of the next proposition which gives a universal property defining the graph product. We retain the notation of this section.

PROPOSITION 1.6. Let $N$ be a monoid and suppose that for each $v \in V$ there is $a$ homomorphism $\varphi_{v}: M_{v} \rightarrow N$ such that

$$
\left(x \varphi_{v}\right)\left(y \varphi_{u}\right)=\left(y \varphi_{u}\right)\left(x \varphi_{v}\right) \quad \text { for all }(u, v) \in E \text { and all } x \in M_{v}, y \in M_{u} .
$$

Put $M=\Gamma_{v \in V} M_{v}$. Then there is a unique homomorphism $\varphi: M \rightarrow N$ such that $x \varphi=x \varphi_{v}$ for all $x \in M_{v}$ and all $v \in V$.

Proof. For each $v \in V$, let $\left\langle A_{v} \mid R_{v}\right\rangle$ be a presentation for $M_{v}$, and let $\langle A \mid R\rangle$ be the presentation for $M$ as at the beginning of the section. Let $\theta: A \rightarrow N$ be the function given by $a \theta=a \varphi_{v}$ where $M_{v}$ is the unique monoid containing $a$. Since each $\varphi_{v}$ is a homomorphism, $\theta$ respects the relations in each $R_{v}$, and, by hypothesis, $\theta$ also respects all the other relations in $R$. Hence there is a unique homomorphism $\varphi: M \rightarrow N$ which restricts to $\theta$ on $A$ and hence to $\varphi_{v}$ on each $M_{v}$.

An immediate consequence is the first part of the following result. 
Proposition 1.7. Let $\Gamma$ be a graph, $V$ be its set of vertices and $\left\{M_{v}\right\}_{v \in V}$ and $\left\{N_{v}\right\}_{v \in V}$ be families of monoids. Let $M=\Gamma_{v \in V} M_{v}$ and $N=\Gamma_{v \in V} N_{v}$. Then, given homomorphisms $\varphi_{v}: M_{v} \rightarrow N_{v}$ for each $v \in V$, there is a unique homomorphism $\varphi: M \rightarrow N$ such that $m_{v} \varphi=m_{v} \varphi_{v}$ for all $v \in V$.

Moreover, if each $\varphi_{v}$ is injective, then so is $\varphi$.

PROOF. All that remains is to prove the final paragraph. Let $a, b \in M$ with $a \varphi=b \varphi$ and suppose that $a, b$ have reduced expressions $a_{1} \circ \cdots \circ a_{m}$ and $b_{1} \circ \cdots \circ b_{n}$ respectively, where $a_{i} \in M_{u_{i}}$ and $b_{j} \in M_{v_{j}}$. Then

$$
\left(a_{1} \varphi_{u_{1}}\right) \ldots\left(a_{m} \varphi_{u_{m}}\right)=a \varphi=b \varphi=\left(b_{1} \varphi_{v_{1}}\right) \ldots\left(b_{n} \varphi_{v_{n}}\right)
$$

and, since the $\varphi_{v}$ are injective, both $\left(a_{1} \varphi_{u_{1}}\right) \circ \cdots \circ\left(a_{m} \varphi_{u_{m}}\right)$ and $\left(b_{1} \varphi_{v_{1}}\right) \circ \cdots \circ$ $\left(b_{n} \varphi_{v_{n}}\right)$ are reduced expressions for $a \varphi$. Hence they are shuffle equivalent so that $m=n$ and, for some permutation $\sigma, a_{i} \varphi_{u_{i}}=b_{i \sigma} \varphi_{v_{i \sigma}}$ for all $i$. Since im $\varphi_{v} \subseteq N_{v}$ for all $v$, we see that $u_{i}=v_{i \sigma}$ for each $i$, and so $a_{i}=b_{i \sigma}$ since $\varphi_{u_{i}}$ is injective. It is now clear that $a_{1} \circ \cdots \circ a_{m}$ and $b_{1} \circ \cdots \circ b_{n}$ are shuffle equivalent so that $a=b$ and hence $\varphi$ is injective.

The following corollary, which can also be easily proved directly, is now immediate.

COROLLARY 1.8. Let $\Gamma$ be a graph with vertex set $V$. If, for each $v \in V$, the monoid $M_{v}$ is embeddable in a group $G_{v}$, then the graph product $\Gamma M_{v}$ is embeddable in the group $\Gamma G_{v}$.

In the next section we use ideas about inverse hulls to demonstrate another result about the closure of a class of right cancellative monoids under graph products. Specifically, we consider right cancellative monoids which satisfy the condition that the intersection of two principal left ideals is either principal or empty. A right cancellative monoid satisfying this condition is called a left LCM monoid. We show that a graph product of left LCM monoids is again a left LCM monoid.

The reason for the terminology, which is borrowed from ring theory, is that the defining condition may also be expressed in terms of divisibility. For a right cancellative monoid $C$ and $a, b \in C$, we say that $a$ is a left multiple of $b$ (and that $b$ is a right factor or divisor of $a$ ) if $a=c b$ for some $c \in C$. If $m$ is is a left multiple of both $b$ and $d$, we say it is a common left multiple of these elements, and such a common left multiple $m$ is a least common left multiple of $b$ and $d$ if every common left multiple of $b$ and $d$ is a left multiple of $m$. Equivalently, $m$ is a least common left multiple of $b$ and $d$ if and only if

$$
C b \cap C d=C m \text {. }
$$

Least common left multiples are sometimes known as left least common multiples. We note that a left LCM monoid is a right cancellative monoid in which any two elements having a common left multiple have a least common left multiple. 
In ring theory (see [1]), an integral domain (not necessarily commutative) is called a left LCM domain if the intersection of any two principal left ideals is principal. Thus an integral domain $R$ is a left LCM domain if and only if the cancellative monoid of its nonzero elements is a left LCM monoid.

Similarly, one defines common right factors and highest common right factors. An element $d$ of $C$ is a highest common right factor of $a$ and $b$ in $C$ if and only if $C d$ is the least upper bound of $C a$ and $C b$ in the partially ordered set of principal left ideals of $C$.

We remark that least common left multiples and highest common right factors are not uniquely determined in general, being defined only up to left multiplication by a unit.

If $C$ is actually cancellative, common right multiple, common left factor, least common right multiple and highest common left factor are defined symmetrically.

Examples of right cancellative LCM monoids abound: the right locally Garside monoids of Dehornoy [10] which, as he points out, include all Artin monoids and all Garside monoids; from ring theory, we have already mentioned the multiplicative monoid of nonzero elements of any LCM domain. Examples of LCM monoids which are right cancellative but not left cancellative are provided by principal left ideal right cancellative monoids; specific examples are the monoids of ordinal numbers less than $\omega^{\alpha}$ (where $\alpha$ is any ordinal number greater than 1) under the dual of the usual operation of ordinal addition.

\section{Inverse hulls}

To any right cancellative monoid $C$, one can associate an inverse monoid called the inverse hull of $C$. Before giving the definition we recall some of the basic concepts of inverse monoids. For more on the general theory of inverse monoids see [16, Ch. 5] and [18].

An inverse monoid is a monoid $M$ such that, for all $a \in M$, there is a unique $b \in M$ such that $a b a=a$ and $b a b=b$. The element $b$ is the inverse of $a$ and is denoted by $a^{-1}$. It is worth noting that $\left(a^{-1}\right)^{-1}=a$ and $(a b)^{-1}=b^{-1} a^{-1}$ for all $a, b \in M$. The set of idempotents $E(M)$ of $M$ forms a commutative submonoid, referred to as the semilattice of idempotents of $M$. In fact, a monoid $M$ is an inverse monoid if and only if $E(M)$ is a commutative submonoid and, for every $a \in M$, there is an element $b \in M$ such that $a b a=a$ (that is, $M$ is regular).

An inverse submonoid of an inverse monoid $M$ is simply a submonoid $N$ closed under taking inverses.

For a nonempty set $X$, a partial permutation is a bijection $\sigma: Y \rightarrow Z$ for some subsets $Y, Z$ of $X$. We allow $Y$ and $Z$ to be empty so that the empty function is regarded as a partial permutation. The set of all partial permutations of $X$ is made into a monoid by using the usual rule for composition of partial functions; it is called the symmetric inverse monoid on $X$ and denoted by $\mathscr{I}_{X}$. That it is an inverse monoid follows from the fact that if $\sigma$ is a partial permutation of $X$, then so is its inverse (as a 
function) $\sigma^{-1}$, and this is the inverse of $\sigma$ in $\mathscr{I}_{X}$ in the sense above. The idempotents of $\mathscr{I}_{X}$ are the partial identities $\varepsilon_{Y}$ for all subsets $Y$ of $X$ where $\varepsilon_{Y}$ is the identity map on the subset $Y$. It is clear that, for $Y, Z \subseteq X, \varepsilon_{Y} \varepsilon_{Z}=\varepsilon_{Y \cap Z}$ and hence that $E\left(\mathscr{I}_{X}\right)$ is isomorphic to the Boolean algebra of all subsets of $X$.

The concept of an inverse hull was introduced by Rees [28] to give an alternative proof of Ore's theorem about the existence of a group of fractions of a left (or right) Ore cancellative monoid $C$. The name was introduced in [7], where the inverse hull of a right cancellative semigroup $C$ is defined. A detailed study of the inverse hull is carried out in [5] where the authors use a definition slightly different from that in [7]. However, the two definitions coincide in the case of inverse hulls of right cancellative monoids, the only case that we consider.

After defining what we mean by an inverse hull and recalling some general results, we show that a graph product of left LCM monoids is also a left LCM monoid, and continue by finding a presentation for the inverse hull of a such a graph product in terms of presentations for its constituent monoids. As a special case we obtain a presentation of the inverse hull of a graph monoid.

2.1. Generalities about inverse hulls As well as being significant in the question of embeddability in a group, the inverse hull of a right cancellative semigroup is also important in describing the structure of bisimple, 0-bisimple, simple and 0-simple inverse semigroups.

Let $C$ be a right cancellative monoid. For an element $a$ of $C$, the mapping $\rho_{a}$ with domain $C$ defined by

$$
x \rho_{a}=x a
$$

is the inner right translation of $C$ determined by $a$. It is injective since $C$ is right cancellative, and so it can be regarded as a member of $\mathscr{I}_{C}$. The inverse submonoid of $\mathscr{I}_{C}$ generated by all the inner right translations of $C$ is the inverse hull $\mathrm{IH}(C)$ of $C$. The inverse of $\rho_{a}$ is, of course, the partial map $\rho_{a}^{-1}: C a \rightarrow C$, so if $C$ is not a group, then $I H(C)$ contains maps which are not total.

The mapping $\eta: C \rightarrow I H(C)$ given by $a \eta=\rho_{a}$ is an embedding of $C$ into $I H(C)$. Moreover, $C \eta$ is the right unit subsemigroup of $I H(C)$, that is, it consists of those elements $\rho \in I H(C)$ for which there is an element $\tau$ with $\rho \tau=1_{C}$. The group of units of $I H(C)$ is $G \eta$, where $G$ is the group of units of $C$. The left unit submonoid $L$ of $I H(C)$ consists of the elements $\rho_{c}^{-1}$ for $c \in C$. For notational convenience, we introduce a left cancellative monoid $C^{-1}$ containing $G$ as its group of units and such that there is an anti-isomorphism $c \mapsto c^{-1}$ from $C$ to $C^{-1}$. Here, if $c \in G$, then $c^{-1}$ is its inverse in $G$, and if $c \notin G$, then $c^{-1}$ is a new symbol. We can now extend $\eta$ from $G$ to an isomorphism, also denoted by $\eta$, from $C^{-1}$ to $L$ given by $c^{-1} \eta=\rho_{c}^{-1}$.

We remark that if $C$ is a group, then every inner right translation is a permutation of $C$ and $\eta$ is just the Cayley representation of $C$.

The empty mapping $\emptyset$ is sometimes a member of $I H(C)$. When it is, it is the zero of $I H(C)$. For ease of expression of some results, we often state them in terms of $I H^{0}(C)$, where we define $I H^{0}(C)$ to be the submonoid $I H(C) \cup\{\varnothing\}$ of $\mathscr{I}_{C}$. 
Clearly, if $a_{1}, \ldots, a_{n}, b_{1}, \ldots, b_{n}$ are elements of $C$, then $\rho=\rho_{a_{1}} \rho_{b_{1}}^{-1} \ldots \rho_{a_{n}} \rho_{b_{n}}^{-1}$ is a member of $I H(C)$. It is easy to verify that every element of $I H(C)$ can be expressed in this way (see [5, Lemma 2.5]) using the fact that if $a, b \in C$, then $\rho_{a} \rho_{b}=\rho_{a b}$ and $\rho_{a}^{-1} \rho_{b}^{-1}=\rho_{b a}^{-1}$. Thus every element can be written in the form $\left(a_{1} \eta\right)\left(b_{1}^{-1} \eta\right) \ldots\left(a_{n} \eta\right)\left(b_{n}^{-1} \eta\right)$.

It is noted in [7] that the inverse hull of an infinite cyclic monoid $\{x\}^{*}$ is the bicyclic monoid. This example was generalized by Nivat and Perrot in [26] where they introduced polycyclic monoids as the inverse hulls of free monoids. They give several characterizations of polycyclic monoids and, in particular, show that the polycyclic monoid $P_{X}$ on a set $X$ with more than one element has the following presentation as a monoid with zero:

$$
\left.\left\langle X \cup X^{-1}\right| x x^{-1}=1, x y^{-1}=0 \text { for } x \neq y(x, y \in X)\right\rangle .
$$

More information on polycyclic monoids can be found in [18, Ch. 9] and [25].

An independent study of the inverse hull of the free monoid on an arbitrary nonempty set $X$ was carried out in [17] where Knox describes it as a Rees quotient of a semidirect product of a semilattice by the free group on $X$.

Further examples of inverse hulls are calculated in [23].

We recall that a compatible partial order called the natural partial order is defined on any inverse semigroup $S$ by the rule that $a \leqslant b$ if $a=e b$ for some idempotent $e$. For later use, we characterize this relation between certain elements of an inverse hull in the following well-known lemma. See [19] for a version of this and its corollary.

LEMMA 2.1. Let $C$ be a right cancellative monoid and let $a, b, c, d \in C$. Then in $\operatorname{IH}(C)$,

$$
\rho_{a}^{-1} \rho_{b} \leqslant \rho_{c}^{-1} \rho_{d} \quad \text { if and only if } a=x c \text { and } b=x d \text { for some } x \in C .
$$

PROOF. If $\rho_{a}^{-1} \rho_{b} \leqslant \rho_{c}^{-1} \rho_{d}$, then $a \in \operatorname{dom} \rho_{a}^{-1} \rho_{b}$, so $a \in \operatorname{dom} \rho_{c}^{-1} \rho_{d}$, that is, $a \in C c$, say $a=x c$. Then

$$
b=a \rho_{a}^{-1} \rho_{b}=a \rho_{c}^{-1} \rho_{d}=x d .
$$

Conversely,

$$
\rho_{a}^{-1} \rho_{b}=\rho_{c}^{-1} \rho_{x}^{-1} \rho_{x} \rho_{d} \leqslant \rho_{c}^{-1} \rho_{d} .
$$

Corollary 2.2. Let $C$ be a right cancellative monoid and let $a, b, c, d \in C$. Then in $\operatorname{IH}(C)$,

$$
\rho_{a}^{-1} \rho_{b}=\rho_{c}^{-1} \rho_{d} \quad \text { if and only if } a=u c \text { and } b=u d \text { for some unit } u \in C .
$$

Proof. By Lemma 2.1, there are elements $x, y \in C$ such that $a=x c, b=x d, c=y a$ and $d=y b$. Hence $a=x y a$ and, by right cancellation, $1=x y$. It follows that $x$ and $y$ are units. 
Recall that in any monoid $M$, Green's relation $\mathscr{R}$ is defined by the rule that $a \mathscr{R} b$ if and only if $a M=b M$. The relation $\mathscr{L}$ is the left-right dual of $\mathscr{R}$; we define $\mathscr{H}=\mathscr{R} \cap \mathscr{L}$ and $\mathscr{D}=\mathscr{R} \vee \mathscr{L}$. In fact, by [16, Proposition 2.1.3], $\mathscr{D}=$ $\mathscr{R} \circ \mathscr{L}=\mathscr{L} \circ \mathscr{R}$. Finally, $a \mathscr{J} b$ if and only if $M a M=M b M$. In an inverse monoid, $a \mathscr{R} b$ if and only if $a a^{-1}=b b^{-1}$ and, similarly, $a \mathscr{L} b$ if and only if $a^{-1} a=b^{-1} b$. In $\mathscr{I}_{X}$, we have $\rho \mathscr{R} \sigma$ if and only if $\operatorname{dom} \rho=\operatorname{dom} \sigma$, and $\rho \mathscr{L} \sigma$ if and only if $\operatorname{im} \rho=\operatorname{im} \sigma$ [16, Exercise 5.11.2]. The following lemma thus follows immediately from [18, Proposition 3.2.11].

LEMMA 2.3. Let $C$ be a right cancellative monoid. Then, for elements $\rho, \sigma$ of $I H^{0}(C)$ :

(1) $\rho \mathscr{R} \sigma$ in $I H^{0}(C)$ if and only if $\operatorname{dom} \rho=\operatorname{dom} \sigma$;

(2) $\rho \mathscr{L} \sigma$ in $I H^{0}(C)$ if and only if im $\rho=\operatorname{im} \sigma$.

We mention that $\mathscr{L}$ is a right congruence and $\mathscr{R}$ is a left congruence. More information on Green's relations can be found in [16, 18]. Finally, an inverse monoid (or semigroup) is 0-bisimple if all its nonzero elements are $\mathscr{D}$-related; it is bisimple if all its elements are $\mathscr{D}$-related. Thus if $a, b$ are nonzero elements of a 0-bisimple inverse monoid $M$, then there are elements $c, d \in M$ such that $a \mathscr{L} c \mathscr{R} b$ and $a \mathscr{R} d \mathscr{L} b$.

It is pointed out in [26] that the equivalence of (1) and (3) in the next proposition can be obtained by slightly modifying the theory of Clifford [6]. A proof of the whole result can be extracted from [21], but for the convenience of the reader and completeness we give an elementary proof.

PROPOSITION 2.4. The following are equivalent for a right cancellative monoid $C$ :

(1) $I H^{0}(C)$ is 0-bisimple;

(2) the domain of each nonzero element of $I^{0}(C)$ is a principal left ideal;

(3) C is a left LCM monoid;

(4) every nonzero element of $I H^{0}(C)$ can be written in the form $\rho_{c}^{-1} \rho_{d}$ for some $c, d \in C$.

Proof. Suppose that (1) holds, and let $\rho$ be a nonzero element of $I H^{0}(C)$. Then $\rho$ is $\mathscr{D}$-related to the identity, and so $\mathscr{R}$-related to an element $\sigma$ of the left unit submonoid. Hence $\operatorname{dom} \rho=\operatorname{dom} \sigma$ and, since $\sigma=\rho_{a}^{-1}$ for some $a \in C$, $\operatorname{dom} \rho=C a$ so that (2) holds.

If (2) holds, and $a, b \in C$, then since $C a \cap C b$ is the domain of $\rho_{a}^{-1} \rho_{a} \rho_{b}^{-1} \rho_{b}$, we see that $C a \cap C b$ is either principal or empty. Thus (3) holds.

Now suppose that (3) holds and let $\rho$ be a nonzero element of $I H^{0}(C)$. We have noted that $\rho=\rho_{a_{1}} \rho_{b_{1}}^{-1} \ldots \rho_{a_{n}} \rho_{b_{n}}^{-1}$ for some $a_{i}, b_{i} \in C$, and so it is enough to show that if $c, d \in C$ and $\rho_{c} \rho_{d}^{-1}$ is nonzero, then $\rho_{c} \rho_{d}^{-1}=\rho_{a}^{-1} \rho_{b}$ for some $a, b \in C$. Now the domain of $\rho_{c} \rho_{d}^{-1}$ is $(C c \cap C d) \rho_{c}^{-1}$, and, by assumption, $C c \cap C d=C s$ for some $s \in C$. Thus $s=r c=t d$ for some $r, t \in C$ and an easy calculation shows that $\rho_{c} \rho_{d}^{-1}=\rho_{r}^{-1} \rho_{t}$. 
Finally, if (4) holds, let $\rho=\rho_{a}^{-1} \rho_{b}$ be a nonzero element of $I H^{0}(C)$. Now $\rho_{a}^{-1}$ is $\mathscr{L}$-related to the identity, and since $\mathscr{L}$ is a right congruence, we get $\rho \mathscr{L} \rho_{b}$. But $\rho_{b} \mathscr{R} 1$, so $\rho$ is $\mathscr{D}$-related to the identity, and (1) follows.

It is worth noting that if $C$ is a left LCM monoid, then the product of two nonzero elements in $I H^{0}(C)$ is given by

$$
\left(\rho_{a}^{-1} \rho_{b}\right)\left(\rho_{c}^{-1} \rho_{d}\right)= \begin{cases}0 & \text { if } C b \cap C c=\emptyset, \\ \rho_{s a}^{-1} \rho_{t d} & \text { if } C b \cap C c=C s b=C t c .\end{cases}
$$

Although it is not relevant to the present paper, it is worth noting that every 0-bisimple inverse monoid $M$ is isomorphic to $I H^{0}(C)$ where $C$ is the right unit submonoid of $M$ [26], so that the preceding proposition applies to all such monoids. We make use of the proposition to prove the next theorem, for which we also need the following lemma.

LEMMA 2.5. Let $\Gamma=(V, E)$ be a graph and, for each $v \in V$, let $C_{v}$ be a right cancellative monoid and $C=\Gamma_{v \in V} C_{v}$. Let $c, d$ be nonunits in $C_{v}, C_{u}$ respectively, where $(u, v) \in E$. Then

$$
C c \cap C d=C c d .
$$

Proof. Since $(u, v) \in E$, we have $c d=d c$ so that $C c d \subseteq C c \cap C d$. Now suppose that $a \in C c \cap C d$ so that $a=s c=t d$ for some $s, t \in C$. By Lemma 1.4, $a$ has final $v$-component $c^{\prime} c$ and final $u$ component $d^{\prime} d$, where $c^{\prime}$ is the final $v$-component of $s$ and $d^{\prime}$ is the final $u$-component of $t$. Neither $c^{\prime} c$ nor $d^{\prime} d$ can be 1 since $c, d$ are not units. Thus $a$ has reduced expressions $x_{1} \circ \cdots \circ x_{n} \circ\left(c^{\prime} c\right)$ and $y_{1} \circ \cdots \circ y_{n} \circ\left(d^{\prime} d\right)$ which, by Theorem 1.1, must be shuffle equivalent. Hence one of the $x_{i}$, say $x_{j}$, must be $d^{\prime} d$ and one can shuffle it to the end to obtain a reduced expression

$$
x_{1} \circ \cdots \circ x_{j-1} \circ x_{j+1} \circ \cdots \circ x_{n} \circ\left(c^{\prime} c\right) \circ\left(d^{\prime} d\right)
$$

for $a$. Hence $a=x_{1} \ldots x_{j-1} x_{j+1} \ldots x_{n}\left(c^{\prime} c\right)\left(d^{\prime} d\right)$ and, since $c \in C_{v}, d^{\prime} \in C_{u}$ so that $c d^{\prime}=d^{\prime} c($ as $(u, v) \in E)$,

$$
a=x_{1} \ldots x_{j-1} x_{j+1} \ldots x_{n} c^{\prime} d^{\prime} c d \in C c d,
$$

completing the proof.

THEOREM 2.6. Let $\Gamma=(V, E)$ be a graph and, for each $v \in V$, let $C_{v}$ be a left LCM monoid. Then the graph product $C=\Gamma_{v \in V} C_{v}$ is also a left LCM monoid.

PRoOF. $C$ is right cancellative by Theorem 1.5. To prove that $C$ is a left LCM monoid, we show that every nonzero element of $I H^{0}(C)$ can be written in the form $\rho_{a}^{-1} \rho_{b}$ for some $a, b \in C$, and appeal to Proposition 2.4.

We claim that if $c, d \in C$ and $\tau=\rho_{c} \rho_{d}^{-1}$ is nonzero, then $\tau=\rho_{a}^{-1} \rho_{b}$ for some $a, b \in C$. The result follows from this claim and our earlier observation that every nonzero element of $I H^{0}(C)$ can be written in the form $\rho_{a_{1}} \rho_{b_{1}}^{-1} \ldots \rho_{a_{n}} \rho_{b_{n}}^{-1}$. 
We note that the claim is true if one of $c, d$ is a unit: if $r=c^{-1}$ exists, then

$$
\tau=\rho_{r^{-1}} \rho_{d}^{-1}=\rho_{r}^{-1} \rho_{d}^{-1}=\rho_{d r}^{-1}=\rho_{d r}^{-1} \rho_{1},
$$

and if $d$ is a unit, then

$$
\rho_{c} \rho_{d}^{-1}=\rho_{c} \rho_{d^{-1}}=\rho_{c d^{-1}}=\rho_{1}^{-1} \rho_{c d^{-1}}
$$

We now assume that $c, d$ are both nonunits and continue by proving the claim in the case where they both have length 1 , so that $c \in C_{v}$ for some $v \in V$. If $d \in C_{v}$, then $\tau=$ $\rho_{a}^{-1} \rho_{b}$ since $C_{v}$ is a left LCM monoid. Let $d \in C_{u}$ with $u \neq v$. If $(u, v) \notin E$, then no reduced expression ending in $c$ is shuffle equivalent to one ending in $d$, and it follows that $C c \cap C d=\emptyset$. Thus $\tau=\emptyset$, a contradiction. Hence $(u, v) \in E$ so that $c d=d c$. By Lemma 2.5, $C c \cap C d=C c d$. It follows that dom $\rho_{c} \rho_{d}^{-1}=C d=\operatorname{dom} \rho_{d}^{-1} \rho_{c}$, and it is easily verified that $\rho_{c} \rho_{d}^{-1}=\rho_{d}^{-1} \rho_{c}$. Hence the claim holds for all $c$ and $d$ of length 1 ; in fact $\rho_{c} \rho_{d}^{-1}=\rho_{a}^{-1} \rho_{b}$, where $a$ and $b$ also have length 1 .

To complete the proof, let $c, d \in C$ have reduced expressions $c_{1} \circ \cdots \circ c_{h}$ and $d_{1} \circ \cdots \circ d_{k}$ so that $\rho_{c} \rho_{d}^{-1}=\rho_{c_{1}} \ldots \rho_{c_{h}} \rho_{d_{1}}^{-1} \ldots \rho_{d_{k}}^{-1}$. Now apply the length 1 case repeatedly.

In the next lemma we compare intersections of principal left ideals in the graph product and in its component monoids.

LEMMA 2.7. Let $\Gamma=(V, E)$ be a graph and, for each $v \in V$, let $C_{v}$ be a left LCM monoid and let $C=\Gamma_{v \in V} C_{v}$. If $x, y \in C_{v}$ for some $v \in V$, then

$$
C_{v} x \cap C_{v} y=\varnothing \quad \text { if and only if } C x \cap C y=\emptyset .
$$

Moreover, if $C_{v} x \cap C_{v} y=C_{v} z$, then $C x \cap C y=C z$.

Proof. Clearly, if $C x \cap C y=\emptyset$, then $C_{v} x \cap C_{v} y=\emptyset$. Conversely, suppose that $a x=b y$ for some $a, b \in C$. Let $a$ and $b$ have final $v$-components $c$ and $d$, respectively. Then by Lemma 1.4, ax has final $v$-component $c x$ and by has final $v$-component $d y$. But $a x=b y$ so, by Proposition 1.3, $c x=b y \in C_{v} x \cap C_{v} y$.

Suppose that $C_{v} x \cap C_{v} y=C_{v} z$; then certainly $C z \subseteq C x \cap C y$. If $r=a x=b y$ for some $a, b \in C$ then, applying Lemma 1.4 and Proposition 1.3 again, we see that $r$ has final $v$-component $c x=d y$ where $c$ and $d$ are the final $v$-components of $a$ and $b$, respectively. Thus $c x \in C_{v} x \cap C_{v} y$ so $c x=m z$ for some $m \in C_{v}$, and if $r^{\prime}$ is the final $v$-complement of $r$, then $r=r^{\prime} m z \in C z$ as required.

We are now in a position to prove the following result which will be important in the next subsection.

Proposition 2.8. If $C$ is the graph product $\Gamma_{v \in V} C_{v}$ of left $L C M$ monoids $C_{v}$, then, for each $v \in V$, the inverse hull $\mathrm{IH}^{0}\left(C_{v}\right)$ is embedded in $\mathrm{IH}^{0}(C)$. 
PRoof. For $x \in C_{v}$ denote the inner right translations of $C_{v}$ and $C$ determined by $x$ by $\rho_{x}$ and $\delta_{x}$, respectively. Nonzero elements of $I H^{0}\left(C_{v}\right)$ have the form $\rho_{x}^{-1} \rho_{y}$ and so we can define $\theta: I H^{0}\left(C_{v}\right) \rightarrow I H^{0}(C)$ by $0 \theta=0$ and $\left(\rho_{x}^{-1} \rho_{y}\right) \theta=\delta_{x}^{-1} \delta_{y}$.

To see that $\theta$ is well defined, suppose that $\rho_{x}^{-1} \rho_{y}=\rho_{z}^{-1} \rho_{t}$. Then by Corollary 2.2, $x=u z$ and $y=u t$ for some unit $u$ of $C_{v}$. Certainly $u$ is a unit of $C$, so $\delta_{x}^{-1} \delta_{y}=\delta_{z}^{-1} \delta_{t}$ as required.

To see that $\theta$ is injective, suppose that $\delta_{x}^{-1} \delta_{y}=\delta_{z}^{-1} \delta_{t}$, where $x, y, z, t \in C_{v}$. Then by Corollary 2.2, $x=q z$ and $y=q t$ for some unit $q$ of $C$. By Corollary 1.2, $C_{v}$ is unitary in $C$, and since $q t, t \in C_{v}$, we have $q \in C_{v}$. It is easy to see that $q^{-1}$ is also in $C_{v}$, so that $q$ is a unit of $C_{v}$ and so $\rho_{x}^{-1} \rho_{y}=\rho_{z}^{-1} \rho_{t}$ as required.

Finally, we show that $\theta$ is a homomorphism. Let $\rho_{x}^{-1} \rho_{y}, \rho_{z}^{-1} \rho_{t}$ be elements of $I H^{0}\left(C_{v}\right)$.

If $C_{v} y \cap C_{v} z=\emptyset$ then, by Lemma 2.7, $C y \cap C z=\emptyset$. From the rule for multiplication following Proposition 2.4, $\left(\rho_{x}^{-1} \rho_{y}\right)\left(\rho_{z}^{-1} \rho_{t}\right)=0$ and, since, by Theorem 2.6, $C$ is left LCM, also $\left(\delta_{x}^{-1} \delta_{y}\right)\left(\delta_{z}^{-1} \delta_{t}\right)=0$.

On the other hand, if $C_{v} y \cap C_{v} z \neq \emptyset$, then since $C_{v}$ is a left LCM monoid, $C_{v} y \cap C_{v} z=C_{v} a$ for some $a \in C_{v}$, say $a=r y=s z$, where $r, s \in C_{v}$. Also, by Lemma 2.7, $C y \cap C z=C a$, and so by the rule for multiplication we see that

$$
\left(\rho_{x}^{-1} \rho_{y}\right)\left(\rho_{z}^{-1} \rho_{t}\right)=\rho_{r x}^{-1} \rho_{s t}^{-1}
$$

and

$$
\left(\delta_{x}^{-1} \delta_{y}\right)\left(\delta_{z}^{-1} \delta_{t}\right)=\delta_{r x}^{-1} \delta_{s t}^{-1} .
$$

It follows that $\theta$ is a homomorphism as required.

2.2. Inverse hulls of graph products of left $\mathbf{L C M}$ monoids Let $\Gamma=(V, E)$ be a graph and $\left\{C_{v}\right\}_{v \in V}$ be a family of left LCM monoids. Let $C=\Gamma_{v \in V} C_{v}$ be the graph product of the $C_{v}$; we have just proved that $C$ is also a left LCM monoid. In this subsection our first goal is to find a presentation (as a monoid with zero) for $\operatorname{IH}^{0}(C)$ in terms of given presentations for the inverse monoids $I H^{0}\left(C_{v}\right)$.

We begin by establishing some notation. Let $D$ be any right cancellative monoid with group of units $G$ and let $Y$ be a symmetric set of monoid generators for $G$ (that is, $y \in Y$ if and only if $\left.y^{-1} \in Y\right)$. We assume that $1 \notin Y$ and take $Y$ to be empty if $G=\{1\}$. Let $X$ be a set of nonunits in $D$ such that $X \cup Y$ generates $D$. Let $X^{-1}=\left\{x^{-1} \mid x \in X\right\}$ be a set disjoint from $X$ such that $x \mapsto x^{-1}$ is a bijection, and $X^{-1} \cup Y$ generates the left cancellative monoid $D^{-1}$ anti-isomorphic to $D$. Since any element of $I H(D)$ can be written in the form $\rho_{a_{1}} \rho_{b_{1}}^{-1} \ldots \rho_{a_{n}} \rho_{b_{n}}^{-1}$, it follows that there is a homomorphism from the free monoid $\left(X \cup X^{-1} \cup Y\right)^{*}$ onto $I H(D)$ sending $x$ to $\rho_{x}, y$ to $\rho_{y}$ and $x^{-1}$ to $\rho_{x}^{-1}$. Thus $I H(D)$ has a presentation of the form $\left\langle X \cup X^{-1} \cup Y \mid R\right\rangle$ for some set of relations $R$. We can also regard $\left\langle X \cup X^{-1} \cup Y \mid R\right\rangle$ as a presentation for $I H^{0}(D)$ in the class of monoids with zero. Since $\rho_{x} \rho_{x}^{-1}=1$ for all $x \in X$, we can assume that $x x^{-1}=1$ is a relation in $R$ for every $x \in X$. Similarly, since $\rho_{y}$ is a unit for all $y \in Y$, we can assume that we have relations $y y^{-1}=1=y^{-1} y$ in $R$ for all $y \in Y$. 
Turning to the graph product $C=\Gamma_{v \in V} C_{v}$, we note that we have a corresponding graph product $C^{-1}=\Gamma_{v \in V} C_{v}^{-1}$ of the left cancellative monoids $C_{v}^{-1}$. Writing $G_{v}$ for the common group of units of $C_{v}$ and $C_{v}^{-1}$, we remark that, by [31, Proposition 7.1], the common group of units of $C$ and $C^{-1}$ is $G=\Gamma_{v \in V} G_{v}$. We also observe that the anti-isomorphisms between the $C_{v}$ and the $C_{v}^{-1}$ extend, by a slight variation of Proposition 1.7, to an anti-isomorphism between $C$ and $C^{-1}$. Now put $S_{v}=I H^{0}\left(C_{v}\right)$ for each $v \in V$, and let $\left\langle X_{v} \cup X_{v}^{-1} \cup Y_{v} \mid R_{v}\right\rangle$ be a presentation for $S_{v}$ of the type described in the previous paragraph. It will be convenient to adopt the following notational convention: $x_{v}, y_{v}$ denote elements of $X_{v}, Y_{v}$, respectively; $t_{v}$ denotes an element of $X_{v} \cup Y_{v}$; and $z_{v}$ denotes any element of $Z_{v}=X_{v} \cup X_{v}^{-1} \cup Y_{v}$.

We now put $X=\bigcup_{v \in V} X_{v}, X^{-1}=\bigcup_{v \in V} X_{v}^{-1}, Y=\bigcup_{v \in V} Y_{v}$, and $Z=X \cup$ $X^{-1} \cup Y$. As in Section 1, we will want to consider the free monoid on $\bigcup_{v \in V} C_{v}$ as well as the free monoid $Z^{*}$. To avoid confusion about the various products, we write $\circ$, as before, for the product in the former free monoid, and $\diamond$ for that in $Z^{*}$.

Next, we introduce several sets of relations amongst words over $X \cup X^{-1} \cup Y$ (and zero) as follows:

(1) $R=\bigcup_{v \in V} R_{v}$;

(2) $N=\left\{x_{v} \diamond y_{u_{1}} \diamond \cdots \diamond y_{u_{m}} \diamond x_{w}^{-1}=0 \mid m \geqslant 0, \forall x_{v} \in X_{v}, x_{w} \in X_{w}\right.$, $y_{u_{i}} \in Y_{u_{i}}$ with $(v, w) \notin E$ and $\left.v \neq w\right\}$;

$$
\text { Com }=\left\{z_{u} \diamond z_{v}=z_{v} \diamond z_{u} \mid \forall z_{u} \in Z_{u}, z_{v} \in Z_{v} \text { with }(u, v) \in E\right\} \text {. }
$$

The polygraph product of the $S_{v}$ is defined to be the monoid $\mathrm{PG}=\mathrm{PG}_{v \in V}\left(S_{v}\right)$ given by the presentation

$$
\langle Z \mid R \cup N \cup \mathrm{Com}\rangle .
$$

There is thus a surjective homomorphism $\zeta: Z^{*} \rightarrow$ PG. For each $v \in V$, the generators and relations of $I H^{0}\left(C_{v}\right)$ are among those for PG and so there is a monoid homomorphism $\psi_{v}$ from $I H^{0}\left(C_{v}\right)$ into PG determined by $\rho_{t_{v}} \psi_{v}=t_{v} \zeta$ and $\rho_{x_{v}}^{-1} \psi_{v}=x_{v}^{-1} \zeta$ for $t_{v} \in X_{v} \cup Y_{v}$ and $x_{v} \in X_{v}$.

The map $\eta_{v}: C_{v} \rightarrow I H_{0}\left(C_{v}\right)$ given by $c \eta_{v}=\rho_{c}$ is an isomorphism of $C_{v}$ with the right unit submonoid of $\mathrm{IH}_{0}\left(C_{v}\right)$. As noted in the preceding subsection, we can also extend $\eta_{v}$ from $G_{v}$ (the group of units of $C_{v}$ ) to the left cancellative monoid $C_{v}^{-1}$ to give an isomorphism onto the left unit submonoid of $I^{0}\left(C_{v}\right)$. Composing $\eta_{v}$ with the restriction of $\psi_{v}$ first to the right unit submonoid of $I H^{0}\left(C_{v}\right)$, then to the left unit submonoid, we obtain monoid homomorphisms from $C_{v}$ and $C_{v}^{-1}$ into PG, both of which we denote by $\theta_{v}$. There is no ambiguity here since these homomorphisms agree on the common group of units of $C_{v}$ and $C_{v}^{-1}$. We observe that if $c_{v}=t_{1} \ldots t_{n}$, where $t_{i} \in X \cup Y$, then

$$
c_{v} \theta_{v}=\left(t_{1} \eta_{v} \psi_{v}\right) \ldots\left(t_{n} \eta_{v} \psi_{v}\right)=\rho_{t_{1}} \psi_{v} \ldots \rho_{t_{n}} \psi_{v}=t_{1} \zeta \ldots t_{n} \zeta=\left(t_{1} \diamond \cdots \diamond t_{n}\right) \zeta
$$

and

$$
c_{v}^{-1} \theta_{v}=\left(t_{n}^{-1} \ldots t_{1}^{-1}\right) \theta_{v}=\rho_{t_{n}}^{-1} \psi_{v} \ldots \rho_{t_{1}}^{-1} \psi_{v}=t_{n}^{-1} \zeta \ldots t_{1}^{-1} \zeta=\left(t_{n}^{-1} \diamond \cdots \diamond t_{1}^{-1}\right) \zeta .
$$


Now by Proposition 1.6 and its dual, there are unique homomorphisms from $C$ into the right unit submonoid of PG, and from $C^{-1}$ into the left unit submonoid of PG which restrict to $\theta_{v}$ on each $C_{v}$ and $C_{v}^{-1}$, respectively. We have noted that the common group of units of $C$ and $C^{-1}$ is $G=\Gamma_{v \in V} G_{v}$, where $G_{v}$ is the common group of units of $C_{v}$ and $C_{v}^{-1}$. As no nonunits are in both $C$ and $C^{-1}$, there is no ambiguity in denoting both homomorphisms by $\theta$.

From the above we see that the squares
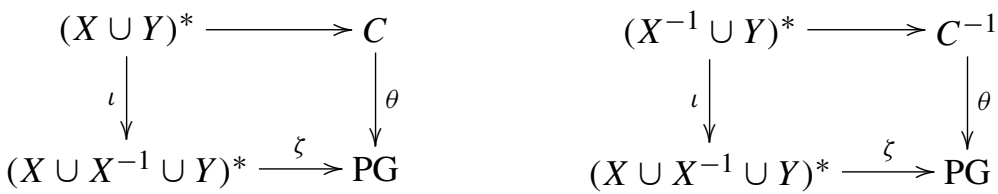

are commutative where $\iota$ is the inclusion map. It follows that every nonzero element of PG can be written in the form $\left(a_{1} \theta\right)\left(b_{1}^{-1} \theta\right) \ldots\left(a_{k} \theta\right)\left(b_{k}^{-1} \theta\right)$, where $a_{i}, b_{i} \in C$. In fact, we can do better than this, as we see in the next lemma.

LEMMA 2.9. Every nonzero element of $\mathrm{PG}=\mathrm{PG}_{v \in V}\left(S_{v}\right)$ can be written in the form $\left(a^{-1} \theta\right)(b \theta)$, where $a, b \in C$.

PROOF. In view of the remark preceding the lemma, it is enough to show that if $c, d \in C$, then either $(c \theta)\left(d^{-1} \theta\right)=0$ or $(c \theta)\left(d^{-1} \theta\right)=\left(a^{-1} \theta\right)(b \theta)$ for some $a, b \in C$. This is clearly true if $c$ or $d$ is a unit of $C$, so we may assume that neither is a unit.

We use induction on the length, as defined in Section 1, of $c$ and $d$. We start by considering $d$ of length 1 , and proving by induction on the length of $c$ that for any $c \in C$, either $(c \theta)\left(d^{-1} \theta\right)=0$ or $(c \theta)\left(d^{-1} \theta\right)=\left(a^{-1} \theta\right)(b \theta)$ for some $a, b \in C$ with $a$ of length 1. First, suppose that $c$ has length 1 . Then $c \in C_{u}, d \in C_{v}$ for some $u, v$. If $u=v$, then

$$
(c \theta)\left(d^{-1} \theta\right)=\left(c \theta_{u}\right)\left(d^{-1} \theta_{u}\right)=\left(\rho_{c} \psi_{u}\right)\left(\rho_{d}^{-1} \psi_{u}\right)=\left(\rho_{c} \rho_{d}^{-1}\right) \psi_{u} .
$$

Since $C_{u}$ is left LCM, we have, by Proposition 2.4, that $\rho_{c} \rho_{d}^{-1}$ is either zero or equal to $\rho_{a}^{-1} \rho_{b}$ for some $a, b \in C_{u}$. Hence, if nonzero,

$$
(c \theta)\left(d^{-1} \theta\right)=\left(\rho_{c} \rho_{d}^{-1}\right) \psi_{u}=\left(\rho_{a}^{-1} \rho_{b}\right) \psi_{u}=\left(\rho_{a}^{-1} \psi_{u}\right)\left(\rho_{b} \psi_{u}\right)=\left(a^{-1} \theta\right)(b \theta) .
$$

If $u \neq v$, let $c=t_{1}^{\prime} \ldots t_{m}^{\prime}$ and $d=t_{1} \ldots t_{n}$, where $t_{i}^{\prime} \in X_{u} \cup Y_{u}$ and $t_{j} \in X_{v} \cup Y_{v}$. If $(u, v) \in E$, then $t_{i}^{\prime} \diamond t_{j}=t_{j} \diamond t_{i}^{\prime}$ is a relation in Com for all $i, j$ and it follows that $(c \theta)\left(d^{-1} \theta\right)=\left(d^{-1} \theta\right)(c \theta)$.

Suppose that $(u, v) \notin E$. Since $c, d$ are nonunits, not all the $t_{i}^{\prime}$ are units and not all the $t_{j}$ are units. Let $h$ and $k$ be the largest integers such $t_{h}^{\prime}$ and $t_{k}$ are nonunits. Then we can write $x_{h}^{\prime}$ for $t_{h}^{\prime}$ and $x_{k}$ for $t_{k}$, and, similarly, we can write $y_{i}^{\prime}$ for $t_{i}^{\prime}$ when $i>h$ and $y_{j}$ for $t_{j}$ when $j>k$. Consider

$$
\left(x_{h}^{\prime} \diamond y_{h+1}^{\prime} \diamond \cdots \diamond y_{m}^{\prime} \diamond y_{n}^{-1} \diamond \cdots \diamond y_{k+1}^{-1} \diamond x_{k+1}^{-1}\right) \zeta .
$$

This element is zero (by virtue of the relations in $N$ ) and so $(c \theta)\left(d^{-1} \theta\right)=0$. 
Thus our claim is true for all $c$ and $d$ of length 1 . Now suppose that, for any $c, d \in C$ with $c$ of length less than $m$ and $d$ of length $1,(c \theta)\left(d^{-1} \theta\right)=0$ or $(c \theta)\left(d^{-1} \theta\right)=\left(a^{-1} \theta\right)(b \theta)$ for some $a, b \in C$ with $a$ of length 1 .

Next, let $c \in C$ have length $m$, let $c_{1} \circ \cdots c_{m}$ be a reduced expression for $c$, and let $d \in C_{v}$. By the current induction assumption, $\left(c_{2} \ldots c_{m} \theta\right)\left(d^{-1} \theta\right)$ is either zero or can be written in the form $\left(a^{-1} \theta\right)(b \theta)$ with $a$ of length 1 . In the former case, it is clear that $(c \theta)\left(d^{-1} \theta\right)=0$. In the latter case, if $(c \theta)\left(d^{-1} \theta\right)$ is nonzero, then

$$
\begin{aligned}
(c \theta)\left(d^{-1} \theta\right) & =\left(\left(c_{1} \ldots c_{m}\right) \theta\right)\left(d^{-1} \theta\right)=\left(c_{1} \theta\right)\left(\left(c_{2} \ldots c_{m}\right) \theta\right)\left(d^{-1} \theta\right) \\
& =\left(c_{1} \theta\right)\left(a^{-1} \theta\right)(b \theta) \\
& =\left(a_{1}^{-1} \theta\right)\left(b_{1} \theta\right)(b \theta)=\left(a_{1}^{-1} \theta\right)\left(\left(b_{1} b\right) \theta\right)
\end{aligned}
$$

where $a_{1}$ has length 1 , using the fact that $c_{1}$ and $a$ both have length 1 .

Thus we have proved our claim that, for any $c, d \in C$ with $d$ of length 1 , either $(c \theta)\left(d^{-1} \theta\right)=0$ or $(c \theta)\left(d^{-1} \theta\right)=\left(a^{-1} \theta\right)(b \theta)$ for some $a, b \in C$ with $a$ of length 1 .

Now assume inductively that for any $c \in C$ and any $d \in C$ of length $n-1$, if $(c \theta)\left(d^{-1} \theta\right) \neq 0$, then $(c \theta)\left(d^{-1} \theta\right)=\left(a^{-1} \theta\right)(b \theta)$ for some $a, b \in C$. Let $d \in C$ have a reduced expression $d_{1} \circ \cdots \circ d_{n}$ so that

$$
\begin{aligned}
(c \theta)\left(d^{-1} \theta\right) & =(c \theta)\left(d_{n}^{-1} \theta\right)\left(\left(d_{n-1}^{-1} \ldots d_{1}^{-1}\right) \theta\right) \\
& \left.=\left(a_{1}^{-1} \theta\right)\left(b_{1} \theta\right)\left(\left(d_{n-1}^{-1} \ldots d_{1}^{-1}\right) \theta\right) \quad \text { for some } a_{1}, b_{1} \in C \text { (by the case for } n=1\right) \\
& =\left(a_{1}^{-1} \theta\right)\left(\left(b_{1} \theta\right)\left(d_{n-1}^{-1} \ldots d_{1}^{-1}\right) \theta\right) \\
& =\left(a_{1}^{-1} \theta\right)\left(a_{2}^{-1} \theta\right)\left(b_{2} \theta\right) \quad \text { for some } a_{2}, b_{2} \in C \text { (by the induction assumption) } \\
& =\left(a_{1}^{-1} a_{2}^{-1}\right) \theta\left(b_{2} \theta\right) \\
& =\left(a^{-1} \theta\right)(b \theta) \quad \text { where } a=a_{2} a_{1} \text { and } b=b_{2} .
\end{aligned}
$$

This completes the proof of the lemma.

We now consider $I H^{0}(C)$. We remind the reader that (as a monoid with zero) each $I H^{0}\left(C_{v}\right)$ is generated by $\left\{\rho_{x_{v}}, \rho_{x_{v}}^{-1}, \rho_{y_{v}}: x_{v} \in X_{v}, y_{v} \in Y_{v}\right\}$ and that $I H^{0}(C)$ is generated by $Q=\left\{\rho_{x}, \rho_{x}^{-1}, \rho_{y} \mid x \in X, y \in Y\right\}$, where $X=\bigcup_{v \in V} X_{v}, X^{-1}=\bigcup_{v \in V} X_{v}^{-1}$ and $Y=\bigcup_{v \in V} Y_{v}$. As before, we also assume that $R_{v}$ is a set of defining relations for $I H^{0}\left(C_{v}\right)$ and put $R=\bigcup_{v \in V} R_{v}$.

LEMMA 2.10. With respect to the generating set $Q$, the relations in $R$ are satisfied by $I H^{0}(C)$.

Proof. By Proposition 2.8, $I H^{0}\left(C_{v}\right)$ is embedded in $I H^{0}(C)$ for all $v \in V$. The relations in $R$ are relations in $R_{v}$ for some $v$, so hold in $I H^{0}\left(C_{v}\right)$ and hence in $I H^{0}(C)$.

LEMMA 2.11. With respect to the generating set $Q$, the relations in $N$ are satisfied by $I H^{0}(C)$. 
PROOF. Suppose that $x_{v} \diamond y_{u_{1}} \diamond \cdots \diamond y_{u_{m}} \diamond x_{w}^{-1}=0$ is a relation in $N$ so that $(v, w) \notin E$ and $v \neq w$. Then in $I H^{0}(C)$,

$$
\operatorname{dom} \rho_{x_{v}} \rho_{y_{u_{1}}} \ldots \rho_{y_{u_{m}}} \rho_{x_{w}}^{-1}=\left(C x_{v} y_{u_{1}} \ldots y_{u_{m}} \cap C x_{w}\right)\left(\rho_{x_{v}} \rho_{y_{u_{1}}} \ldots \rho_{y_{u_{m}}}\right)^{-1} .
$$

Since $x_{v}$ is not a unit and $(v, w) \notin E$, in an expression for an element $a$ of $C x_{v} y_{u_{1}} \ldots y_{u_{m}}$, any amalgamation involving $x_{v}$ produces a nonunit of $C_{v}$, so a nonunit of $C_{w}$ cannot be shuffled to the end of the expression. Hence the final $w$ component of $a$ is a unit. But the final $w$-component of an element of $C x_{w}$ must be a left multiple of $x_{w}$ and hence be a nonunit. It follows from Proposition 1.3 that $C x_{v} y_{u_{1}} \ldots y_{u_{m}} \cap C x_{w}=\emptyset$ and so $\rho_{x_{v}} \rho_{y_{u_{1}}} \ldots \rho_{y_{u_{m}}} \rho_{x_{w}}^{-1}=0$.

LEMMA 2.12. With respect to the generating set $Q$, the relations in Com are satisfied by $I H^{0}(C)$.

PROOF. Following our convention that $t_{u}, x_{u}$ denote arbitrary elements of $X_{u} \cup Y_{u}$ and $X_{u}$ respectively, relations in Com have one of the forms

(i) $t_{u} \diamond t_{v}=t_{v} \diamond t_{u}$,

(ii) $x_{u} \diamond x_{v}^{-1}=x_{v}^{-1} \diamond x_{u}$, or

(iii) $x_{u}^{-1} \diamond x_{v}^{-1}=x_{v}^{-1} \diamond x_{u}^{-1}$,

where $(u, v) \in E$. Relations of the form (i) are satisfied in $I H^{0}(C)$ since

$$
\rho_{t_{u}} \rho_{t_{v}}=\rho_{t_{u} t_{v}}=\rho_{t_{v} t_{u}}=\rho_{t_{v}} \rho_{t_{u}} \text {. }
$$

Consider a relation as in (ii). By Lemma 2.5, $C x_{u} \cap C x_{v}=C x_{u} x_{v}$, and since $x_{u} x_{v}=x_{v} x_{u}$ in $C$,

$$
\operatorname{dom} \rho_{x_{u}} \rho_{x_{v}}^{-1}=\left(\operatorname{im} \rho_{x_{u}} \cap \operatorname{dom} \rho_{x_{v}}^{-1}\right) \rho_{x_{u}}^{-1}=\left(C x_{v} x_{u}\right) \rho_{x_{u}}^{-1}=C x_{v} .
$$

Similarly, we calculate im $\rho_{x_{u}} \rho_{x_{v}}^{-1}=C x_{u}$.

Since im $\rho_{x_{v}}^{-1}=C=\operatorname{dom} \rho_{x_{u}}$, it is easy to see that we also have dom $\rho_{x_{v}}^{-1} \rho_{x_{u}}=C x_{v}$ and $\operatorname{im} \rho_{x_{v}}^{-1} \rho_{x_{u}}=C x_{u}$, and it follows that $\rho_{x_{u}} \rho_{x_{v}}^{-1}=\rho_{x_{v}}^{-1} \rho_{x_{u}}$.

Finally consider a relation of the form (iii). In this case, since $(u, v) \in E$, we also have that $x_{u} \diamond x_{v}=x_{v} \diamond x_{u}$ is a relation in Com. Hence $\rho_{x_{v}} \rho_{x_{u}}=\rho_{x_{u}} \rho_{x_{v}}$ follows by (i), and since $I H^{0}(C)$ is an inverse monoid,

$$
\rho_{x_{u}}^{-1} \rho_{x_{v}}^{-1}=\left(\rho_{x_{v}} \rho_{x_{u}}\right)^{-1}=\left(\rho_{x_{u}} \rho_{x_{v}}\right)^{-1}=\rho_{x_{v}}^{-1} \rho_{x_{u}}^{-1} .
$$

We now use the lemmas together to obtain the following theorem, in which we retain the notation of this section.

THEOREM 2.13. The monoids $\mathrm{PG}_{v \in V}\left(S_{v}\right)$ and $I H^{0}(C)$ are isomorphic.

Proof. Consider the function $\beta: X \cup X^{-1} \cup Y \rightarrow I H^{0}(C)$ given by $x \beta=\rho_{x}$, $x^{-1} \beta=\rho_{x}^{-1}$ and $y \beta=\rho_{y}$. It follows from Lemmas 2.10-2.12 that $\beta$ extends to a 
homomorphism, again denoted by $\beta$, from PG to $I H^{0}(C)$. Since the latter is generated by $Q$, the homomorphism is surjective.

Let $r, s \in \mathrm{PG}$ and suppose that $r \beta=s \beta$. By Lemma 2.9, $r=\left(a^{-1} \theta\right)(b \theta)$ and $s=\left(c^{-1} \theta\right)(d \theta)$ for some $a, b, c, d \in C$. Hence $\left(\left(a^{-1} \theta\right)(b \theta)\right) \beta=\left(\left(c^{-1} \theta\right)(d \theta)\right) \beta$ so that $\rho_{a}^{-1} \rho_{b}=\rho_{c}^{-1} \rho_{d}$, and hence, by Corollary 2.2, there is a unit $e$ of $C$ such that $c=e a$ and $d=e b$. If $m, n \in C$, then there are correponding elements $m^{-1}, n^{-1}$ in $C^{-1}$ and $(m n)^{-1}=n^{-1} m^{-1}$. Thus, using the fact that $e$ is a unit in $C$,

$$
\begin{aligned}
s & =\left(c^{-1} \theta\right)(d \theta)=\left((e a)^{-1} \theta\right)((e b) \theta) \\
& =\left(a^{-1} e^{-1}\right) \theta(e b) \theta=\left(a^{-1} \theta\right)\left(e^{-1} \theta\right)(e \theta)(b \theta) \\
& =\left(a^{-1} \theta\right)\left(\left(e^{-1} e\right) \theta\right)(b \theta)=\left(a^{-1} \theta\right)(b \theta) \\
& =r .
\end{aligned}
$$

Thus $\beta$ is an isomorphism and the proof is complete.

\section{Polygraph monoids}

Theorem 2.13 gives us a presentation for $I H^{0}(C)$ and also allows us to write the elements of PG in the form $a^{-1} b$ with $a, b \in C$ where $a^{-1} b=c^{-1} d$ if and only if $c=e a$ and $d=e b$ for some unit $e$ of $C$. The presentation simplifies considerably in the case when each $C_{v}$ (and hence also $C$ ) has a trivial group of units, in that $Y=\emptyset$ and consequently

$$
N=\left\{x_{u} \diamond x_{v}^{-1}=0 \mid \forall x_{u} \in X_{u}, x_{v} \in X_{v} \text { with }(u, v) \notin E \text { and } u \neq v\right\} .
$$

Thus we have the presentation

$$
\left\langle X \cup X^{-1} \mid R \cup N \cup \operatorname{Com}\right\rangle
$$

for $I H^{0}(C)$.

A particular instance of this is when each $C_{v}$ is a free monogenic monoid. Then $S_{v}=I H^{0}\left(C_{v}\right)$ is the bicyclic monoid with zero adjoined, and as a monoid with zero it has the presentation with two generators: $\left\langle x_{v}, x_{v}^{-1} \mid x_{v} x_{v}^{-1}=1\right\rangle$. In this case, the graph product of the $C_{v}$ is a graph monoid $M(\Gamma)$ with presentation

$$
\left.\left\langle x_{v}(v \in V)\right| x_{u} x_{v}=x_{v} x_{u} \text { if }(u, v) \in E\right\rangle .
$$

The monoid $I H^{0}(M(\Gamma))$ is called a polygraph monoid and we denote it by $P(\Gamma)$. Put $X=\left\{x_{v} \mid v \in V\right\}$ and, for $x \in C_{u}, y \in C_{v}$, write $x \sim y$ if $(u, v) \in E$, and, in an abuse of notation, write $x \nsim y$ to mean $u \neq v$ and $(u, v) \notin E$. Then our polygraph monoid has a presentation

$$
\begin{aligned}
\left\langle X \cup X^{-1}\right| x x^{-1} & =1 ; x y^{-1}=0 \text { if } x \nsim y ; \\
x y & \left.=y x, x y^{-1}=y^{-1} x, x^{-1} y^{-1}=y^{-1} x^{-1} \text { if } x \sim y\right\rangle .
\end{aligned}
$$


If $\Gamma$ has no edges, then $M(\Gamma)=X^{*}$ is the free monoid on $X$ and the polygraph monoid $I H^{0}(M(\Gamma))$ is the monoid with presentation

$$
\left.\left\langle X \cup X^{-1}\right| x x^{-1}=1 ; x y^{-1}=0 \text { if } x \neq y\right\rangle,
$$

that is, it is the polycyclic monoid introduced in [26] and studied in, among others, $[17,18,25]$.

Let $P(\Gamma)$ be the polygraph monoid determined by the graph $\Gamma=(V, E)$. Since $P(\Gamma)$ is the inverse hull (with zero adjoined if necessary) of the graph monoid $M(\Gamma)$, it follows from the remarks following Theorem 2.13 that every nonzero element of $P(\Gamma)$ can be written as $a^{-1} b$ for some $a, b \in M(\Gamma)$. Since the identity is the only unit in $M(\Gamma)$ it follows that if $a, b, c, d \in M(\Gamma)$, then $a^{-1} b=c^{-1} d$ if and only if $a=c$ and $b=d$. Thus we may regard the nonzero elements of $P(\Gamma)$ as pairs $(a, b)$ where $a, b \in M(\Gamma)$. With this notation, the product in $P(\Gamma)$ is given by

$$
(a, b)(c, d)= \begin{cases}0 & \text { if } M(\Gamma) b \cap M(\Gamma) c=\emptyset \\ (s a, t d) & \text { if } M(\Gamma) b \cap M(\Gamma) c=M(\Gamma) s b=M(\Gamma) t c .\end{cases}
$$

Proposition 3.1. The monoid $P(\Gamma)$ is a 0-bisimple (bisimple if it has no zero) inverse monoid with

$$
E(P(\Gamma))=\{(a, a) \mid a \in M(\Gamma)\} \cup\{0\}
$$

as its set of idempotents.

PROOF. Since graph monoids are left LCM, Proposition 2.4 gives that $P(\Gamma)$ is a 0 bisimple (bisimple if it has no zero) inverse monoid.

It is easy to verify that any element of the form $(a, a)$ is idempotent. Suppose that $(a, b)(a, b)=(a, b)$. Then $(t a, s b)=(a, b)$ where $M(\Gamma) a \cap M(\Gamma) b=$ $M(\Gamma) s b=M(\Gamma) t a$. Hence, by the criterion for equality, $t a=a$ and $s b=b$ in $M(\Gamma)$ so that $t=s=1$. Thus $M(\Gamma) a=M(\Gamma) b$ and hence $a=b$.

Since $P(\Gamma)$ is 0-bisimple, $\mathscr{D}=\mathscr{J}$ and two elements are $\mathscr{D}$-related if and only if they are both nonzero or both equal to zero. In the next proposition we characterize the other Green relations on $P(\Gamma)$.

Proposition 3.2. For elements $(a, b),(c, d)$ of $P(\Gamma)$ :

(1) $(a, b)^{-1}=(b, a)$;

(2) $(a, b) \mathscr{L}(c, d)$ if and only if $b=d$;

(3) $(a, b) \mathscr{R}(c, d)$ if and only if $a=c$;

(4) $\mathscr{H}$ is trivial.

PROOF. (1) is an easy calculation. In an inverse monoid, elements $s, t$ are $\mathscr{L}$-related if and only if $s^{-1} s=t^{-1} t$. Using this and (1) we see that in $P(\Gamma)$ we have $(a, b) \mathscr{L}(c, d)$ if and only if $b=d$.

The result for $\mathscr{R}$ is similar, and then it follows that $\mathscr{H}$ is trivial. 
We next consider the properties of being $E^{*}$-unitary or strongly $E^{*}$-unitary. For any inverse monoid $S$, the semilattice of idempotents of $S$ is denoted by $E(S)$, and if $S$ has a zero, then $E^{*}(S)$ denotes the set of nonzero idempotents. Recall from Section 1 that a subset $U$ of $S$ is right unitary in $S$ if for $u \in U, s \in S$ we have $s u \in U$ if and only if $s \in U$. There is a dual notion of left unitary, and if $U$ is both left and right unitary, it is said to be unitary in $S$. If $U$ is either $E(S)$ or $E^{*}(S)$, then it is left unitary if and only if it is right unitary. We say that $S$ is $E$-unitary if $E(S)$ is a unitary subset of $S$, and that it is $E^{*}$-unitary [30] (or 0 - $E$-unitary $[18,25]$ ) if $E^{*}(S)$ is a unitary subset of $S$. $E^{*}$-unitary inverse semigroups are discussed in detail in [18, Ch. 9].

A special class of $E^{*}$-unitary inverse semigroups was introduced independently in [3] and [19]. In general, if we adjoin a zero to a semigroup $S$, we denote the semigroup obtained by $S^{0}$. An inverse semigroup $S$ with zero is strongly $E^{*}$-unitary if there is a group $G$ and a function $\theta: S \rightarrow G^{0}$ satisfying:

(1) $a \theta=0$ if and only if $a=0$;

(2) $a \theta=1$ if and only if $a \in E^{*}(S)$;

(3) if $a b \neq 0$, then $(a b) \theta=(a \theta)(b \theta)$.

Condition (1) says that $\theta$ is 0-restricted; conditions (1) and (2) together say that $\theta$ is idempotent pure, that is, the only elements which map to idempotents are idempotents; and condition (3) says that $\theta$ is a prehomomorphism. In general, prehomomorphisms between inverse monoids are defined in terms of the natural order on the monoids, but the general definition is equivalent to condition (3) when the codomain is a group with zero adjoined. Implicit in [3] is the result that an inverse semigroup with zero is strongly $E^{*}$-unitary if and only if it is a Rees quotient of an $E$-unitary inverse semigroup. This was made explicit with an easy proof in [29]. As well as [3] and [29], further information about strongly $E^{*}$-unitary inverse semigroups, including many examples, can be found in the surveys [20] and [22].

We are interested in the connection between strongly $E^{*}$-unitary inverse monoids and embeddability of cancellative monoids in groups. The following result is due to Margolis [24]; we include a proof for completeness.

PROposition 3.3. Let $S$ be a cancellative monoid. Then $S$ is embeddable in a group if and only if $\operatorname{IH}^{0}(S)$ is strongly $E^{*}$-unitary.

ProOf. Suppose first that $S$ is embedded in a group $G$. As noted in Section 2.1, every (nonzero) element $\rho$ of $I H^{0}(S)$ can be expressed as $\rho_{a_{1}} \rho_{b_{1}}^{-1} \ldots \rho_{a_{n}} \rho_{b_{n}}^{-1}$ for some elements $a_{1}, b_{1}, \ldots, a_{n}, b_{n}$ of $S$. Define a mapping $\theta: I H^{0}(S) \rightarrow G^{0}$ by putting $0 \theta=0$ and $\left(\rho_{a_{1}} \rho_{b_{1}}^{-1} \ldots \rho_{a_{n}} \rho_{b_{n}}^{-1}\right) \theta=a_{1} b_{1}^{-1} \ldots a_{n} b_{n}^{-1}$ if $\rho_{a_{1}} \rho_{b_{1}}^{-1} \ldots \rho_{a_{n}} \rho_{b_{n}}^{-1}$ is nonzero.

If $\rho=\rho_{a_{1}} \rho_{b_{1}}^{-1} \ldots \rho_{a_{n}} \rho_{b_{n}}^{-1}=\rho_{c_{1}} \rho_{d_{1}}^{-1} \ldots \rho_{c_{m}} \rho_{d_{m}}^{-1}$ is nonzero, then, for every element $x$ in $\operatorname{dom} \rho$,

$$
x \rho=x \rho_{a_{1}} \rho_{b_{1}}^{-1} \ldots \rho_{a_{n}} \rho_{b_{n}}^{-1}=x \rho_{c_{1}} \rho_{d_{1}}^{-1} \ldots \rho_{c_{m}} \rho_{d_{m}}^{-1},
$$


so that, in $G$,

$$
x \rho=x a_{1} b_{1}^{-1} \ldots a_{n} b_{n}^{-1}=x c_{1} d_{1}^{-1} \ldots c_{m} d_{m}^{-1}
$$

and hence $a_{1} b_{1}^{-1} \ldots a_{n} b_{n}^{-1}=c_{1} d_{1}^{-1} \ldots c_{m} d_{m}^{-1}$. Thus $\theta$ is well defined.

By definition, $\theta$ is 0 -restricted. If $\rho$ is as defined above and $\rho \theta=1$, then $a_{1} b_{1}^{-1} \ldots a_{n} b_{n}^{-1}=1$ and it follows that $x \rho=x$ for all $x \in \operatorname{dom} \rho$ so that $\rho=I_{\operatorname{dom} \rho}$ and $\theta$ is idempotent pure. Finally, it is clear from the definition that if $\rho, \sigma \in I H^{0}(S)$ and $\rho \sigma \neq 0$, then $(\rho \sigma) \theta=(\rho \theta)(\sigma \theta)$ so that $\theta$ is a prehomomorphism. Thus $I^{0}(S)$ is strongly $E^{*}$-unitary.

For the converse, we suppose that $I H^{0}(S)$ is strongly $E^{*}$-unitary and consider a 0-restricted idempotent pure prehomomorphism $\theta: I H^{0}(S) \rightarrow G^{0}$ from $I H^{0}(S)$ to a group $G$ with zero adjoined. For each $a \in S$, we have the element $\rho_{a}$ of $I H(S)$, and since dom $\rho_{a}=S$, it follows that $\rho_{a} \rho_{b}=\rho_{a b}$ for any $a, b \in S$. Since $\theta$ is 0 -restricted, $\rho_{a} \theta \in G$ and

$$
\left(\rho_{a} \theta\right)\left(\rho_{b} \theta\right)=\left(\rho_{a} \rho_{b}\right) \theta=\rho_{a b} \theta .
$$

Hence we can define $\psi: S \rightarrow G$ by $a \psi=\rho_{a} \theta$, and $(a \psi)(b \psi)=(a b) \psi$, that is, $\psi$ is a homomorphism. It is also injective, for if $a \psi=b \psi$, then $\rho_{a} \theta=\rho_{b} \theta$. Now $\rho_{a}^{-1} \rho_{b}$ is a nonzero element of $I H^{0}(S)$, and so

$$
\left(\rho_{a}^{-1} \rho_{b}\right) \theta=\left(\rho_{a}^{-1} \theta\right)\left(\rho_{b} \theta\right)=\left(\rho_{a}^{-1} \theta\right)\left(\rho_{a} \theta\right)=\left(\rho_{a}^{-1} \rho_{a}\right) \theta=1
$$

since $\rho_{a}^{-1} \rho_{a}$ is a nonzero idempotent. But $\theta$ is idempotent pure, so $\rho_{a}^{-1} \rho_{b}$ is an idempotent, that is, it is the identity map on its domain. Hence, for $x \in \operatorname{dom}\left(\rho_{a}^{-1} \rho_{b}\right)$, $x \rho_{a}^{-1} \rho_{b}=x$. Now $x \rho_{a}^{-1}=u$ where $x=u a$ and also $x=u \rho_{b}=u b$ so that $u a=u b$ and $a=b$ by cancellation.

Thus $S$ is embedded in $G$.

It is well known (and a consequence of Corollary 1.8) that there is an embedding $\theta: M(\Gamma) \rightarrow G(\Gamma)$ of the graph monoid $M(\Gamma)$ into the graph group $G(\Gamma)$, and so we have the following corollary.

Corollary 3.4. For any graph $\Gamma$, the polygraph monoid $P(\Gamma)$ is strongly $E^{*}$ unitary.

In the next section, we see that $P(\Gamma)$ has another special property, namely that it is $F^{*}$-inverse.

\section{4. $F^{*}$-inverse 0-bisimple inverse monoids}

Recall that an inverse monoid $S$ is $F^{*}$-inverse if every nonzero element of $S$ is under a unique maximal element in the natural partial order. If $S$ does not have a zero, it is said to be $F$-inverse, and in this case the definition is equivalent to every $\sigma$-class containing a maximum element. (Here $\sigma$ is the minimum group congruence on $S$.) However, we shall use the term $F^{*}$-inverse to include both cases. It is easy to 
verify that every $F^{*}$-inverse monoid is $E^{*}$-unitary. An $F^{*}$-inverse monoid which is also strongly $E^{*}$-unitary is called strongly $F^{*}$-inverse. It follows from Corollary 3.4 and the results of this section that a polygraph monoid is strongly $F^{*}$-inverse.

We find a criterion for a 0-bisimple inverse monoid with cancellative right unit submonoid to be $F^{*}$-inverse in terms of a property of its right unit submonoid. We remark that by a result of Lawson [19], for a 0-bisimple inverse monoid, having a cancellative right unit submonoid is equivalent to being $E^{*}$-unitary.

LEMMA 4.1. Let $C$ be a right cancellative monoid and suppose that $I H^{0}(C)$ is 0 bisimple. If $a, b \in C$ have only units as common left factors, then $\rho_{a}^{-1} \rho_{b}$ is maximal in $I H^{0}(C)$.

PROOF. Since $I H^{0}(C)$ is 0-bisimple, every element has the form $\rho_{a}^{-1} \rho_{b}$ for some $a, b \in C$. The result is now immediate from Lemma 2.1 and its corollary.

If $C$ is a cancellative monoid, we denote the partially ordered set of principal right (left) ideals by $P_{r}(C)\left(P_{\ell}(C)\right)$. From the remarks at the end of Section 1, we see that $P_{r}(C)$ is a join semilattice if and only if every pair of elements has a highest common left factor, and it is a meet semilattice if and only if every pair of elements has a least common right multiple. Corresponding remarks apply to $P_{\ell}(C)$.

Proposition 4.2. Let $C$ be a cancellative monoid and suppose that $I H^{0}(C)$ is 0 bisimple. Then $\mathrm{IH}^{0}(C)$ is $F^{*}$-inverse if and only if $P_{r}(C)$ is a join semilattice.

PROOF. Suppose that every pair of elements of $C$ has a highest common left factor and let $\alpha$ be a nonzero element of $I H^{0}(C)$. Then $\alpha=\rho_{a}^{-1} \rho_{b}$ for some $a, b \in C$. Let $x$ be a highest common left factor of $a$ and $b$, say $a=x c$ and $b=x d$. Then the only common left factors of $c$ and $d$ are units, so, by Lemma 4.1, $\rho_{c}^{-1} \rho_{d}$ is maximal. But $\rho_{a}^{-1} \rho_{b} \leqslant \rho_{c}^{-1} \rho_{d}$ by Lemma 2.1, so $\alpha$ lies beneath a maximal element.

If $\rho_{a}^{-1} \rho_{b} \leqslant \rho_{p}^{-1} \rho_{q}$ for some $p, q \in C$ then, by Lemma 2.1, $a=y p$ and $b=y q$ for some $q \in C$. Hence $x=y z$ for some $z \in C$ so that $a=y p=y z c$ and $b=y q=y z d$. By left cancellation, $p=z c$ and $q=z d$ so that $\rho_{p}^{-1} \rho_{q} \leqslant \rho_{c}^{-1} \rho_{d}$ by Lemma 2.1. Thus $\rho_{c}^{-1} \rho_{d}$ is the unique maximal element above $\rho_{a}^{-1} \rho_{b}$, and $I H^{0}(C)$ is $F^{*}$-inverse.

Conversely, suppose that $I H^{0}(C)$ is $F^{*}$-inverse, and let $a, b \in C$. Then there is a unique maximal element $\rho_{c}^{-1} \rho_{d}$ above $\rho_{a}^{-1} \rho_{b}$. By Lemma 2.1, $a=x c$ and $b=x d$ for some $x \in C$. If $y$ is a common left factor of $a$ and $b$, then $a=y p$ and $b=y q$ for some $p, q \in C$ so that $\rho_{a}^{-1} \rho_{b} \leqslant \rho_{p}^{-1} \rho_{q}$. Now $\rho_{p}^{-1} \rho_{q} \leqslant \alpha$ for some maximal $\alpha$, and, by uniqueness, $\alpha=\rho_{c}^{-1} \rho_{d}$. It follows that $p=z c$ and $q=z d$ for some $z$ so that $x c=a=y z c$, whence $x=y z$ and $y$ is a left factor of $x$. Thus $x$ is a highest common left factor of $a$ and $b$.

An abstract version of this proposition is given in the following result.

Proposition 4.3. Let $S$ be an $E^{*}$-unitary 0-bisimple (E-unitary bisimple) inverse monoid, and let $C$ be its right unit submonoid. Then $S$ is $F^{*}$-inverse ( $F$-inverse) if and only if $P_{r}(C)$ is a join semilattice. 
Proof. Since $S$ is 0-bisimple, the right unit submonoid $C$ of $S$ is a left LCM monoid by [26, Proposition 1], and from the same proposition we have that $S$ is isomorphic to $I H^{0}(C)$. By [19, Theorem 5], $C$ is cancellative so that the result is now immediate by Proposition 4.2.

A Garside monoid is defined to be a cancellative monoid whose only unit is the identity, that is, a lattice with respect to both left and right divisibility, and that satisfies additional finiteness conditions (see, for example, [9]). Such monoids have proved to be important in the study of algebraic and algorithmic properties of braid groups and, more generally, Artin groups of finite type. We note that if $C$ is a Garside monoid, then since the identity is the only unit, regarded as a partially ordered set under left divisibility, $C$ is order-isomorphic to $P_{r}(C)$ under reverse inclusion. Thus $P_{r}(C)$ is a lattice so that $I H(C)$ does not have a zero, and hence the next corollary follows immediately from Propositions 2.4 and 4.2.

Corollary 4.4. The inverse hull of a Garside monoid $C$ is a bisimple F-inverse monoid.

We now turn to Artin monoids. Recall that an Artin monoid is a monoid generated by a nonempty set $X$ that is subject to relations of the form $x y x \ldots=y x y \ldots$, where $x, y \in X$, both sides of a given relation have the same length, and at most one such relation holds for each pair $x, y \in X$. Thus graph monoids are Artin monoids where both sides of each defining relation have length 2. The associated Artin group of a given Artin monoid $A$ is the group given by the presentation of $A$ regarded as a group presentation. Rather than the definition, we use some of the properties of Artin monoids which we now recall. The first three in the list below can be found in [2], the third is also given in [11], and the fourth is from [27]. Let $A$ be an Artin monoid. Then we have the following properties:

(1) $A$ is cancellative;

(2) the intersection of two principal left (right) ideals of $A$ is either empty or principal;

(3) $A$ is left (and right) Ore if and only if it is of finite type;

(4) $A$ embeds in its associated Artin group.

Proposition 4.5. The inverse hull $I H(A)$ of an Artin monoid $A$ is strongly $F^{*}$ inverse.

PROOF. It follows from Proposition 3.3 and item (4) above that $I H(A)$ is strongly $E^{*}$ unitary ( $E$-unitary if $A$ is of finite type). Moreover, we have already noted that (4) of Proposition 2.4 is satisfied. Hence $I H(A)$ is 0-bisimple (bisimple if $A$ is of finite type).

Thus by Proposition 4.2, it is enough to show that any two elements of $A$ have a highest common left factor. This is noted in [2]. The argument is as follows. Since the defining relations of $A$ are homogeneous (that is, the two words in each relation have the same length), it follows that any factor (left or right) of an element $w$ of $A$ has length at most $|w|$. Hence any element of $A$ has only finitely many left factors. 
Let $x_{1}, \ldots, x_{k}$ be the common left factors of two elements $v$ and $w$ of $A$. Then by the right-handed version of item (2),

$$
x_{1} A \cap \cdots \cap x_{k} A=x A
$$

for some $x$. (That is, $x$ is the least common left multiple of $x_{1}, \ldots, x_{k}$.) Now $x$ is a common left factor of $v$ and $w$ (so must be one of the $x_{i}$ ), and is clearly the highest common left factor of $v$ and $w$.

Since a graph monoid is a special type of Artin monoid, we immediately have the following corollary.

COROLlARY 4.6. For a graph $\Gamma$, the polygraph monoid is a strongly $F^{*}$-inverse monoid.

\section{Acknowledgements}

The work reported in this paper was started during a visit by the first author to Carleton University where the second author was conducting research supported by the Leverhulme Trust. The first author would like to thank Benjamin Steinberg for making his visit possible, and the School of Mathematics and Statistics at Carleton for its hospitality. The paper was completed when the second author was an RCUK Academic Fellow at the University of Manchester.

\section{References}

[1] R. A. Beauregard, 'Right LCM domains', Proc. Amer. Math. Soc. 30 (1971), 1-7.

[2] E. Brieskorn and K. Saito, 'Artin-Gruppen und Coxeter-Gruppen', Invent. Math. 17 (1972), 245-271.

[3] S. Bulman-Fleming, J. Fountain and V. Gould, 'Inverse semigroups with zero: covers and their structure', J. Aust. Math. Soc. 67 (1999), 15-30.

[4] R. Charney, 'An introduction to right-angled Artin groups', Geom. Dedicata 125 (2007), 141-158.

[5] A. Cherubini and M. Petrich, 'The inverse hull of right cancellative semigroups', J. Algebra 111 (1987), 74-113.

[6] A. H. Clifford, 'A class of $d$-simple semigroups', Amer. J. Math. 75 (1953), 547-556.

[7] A. H. Clifford and G. B. Preston, The Algebraic Theory of Semigroups, Vol. I (American Mathematical Society, Providence, RI, 1961).

[8] J. Crisp and M. Laca, 'On the Toeplitz algebras of right-angled and finite-type Artin groups', J. Aust. Math. Soc. 72 (2002), 223-245.

[9] P. Dehornoy, 'Groupes de Garside', Ann. Sci. École Norm. Sup. 35 (2002), 267-306.

[10] , 'Alternating normal forms for braids and locally Garside monoids', J. Pure Appl. Algebra 212 (2008), 2413-2439.

[11] P. Deligne, 'Les immeubles des groupes de tresses généralisés', Invent. Math. 17 (1972), 273-302.

[12] V. Diekert, Combinatorics on Traces, Lecture Notes in Computer Science, 454 (Springer, Berlin, 1990).

[13] E. Fohry and D. Kuske, 'On graph products of automatic and biautomatic monoids', Semigroup Forum 72 (2006), 337-352. 
[14] E. R. Green, Graph Products of Groups. PhD Thesis, University of Leeds, 1990.

[15] S. Hermiller and J. Meier, 'Algorithms and geometry for graph products of groups', J. Algebra 171 (1995), 230-257.

[16] J. M. Howie, Fundamentals of Semigroup Theory (Oxford University Press, Oxford, 1995).

[17] N. Knox, 'The inverse hull of the free semigroup on a set X', Semigroup Forum 16 (1978), 345-354.

[18] M. V. Lawson, Inverse Semigroups: The Theory of Partial Symmetries (World Scientific, Singapore, 1998).

[19] - 'The structure of 0-E-unitary inverse semigroups: I. The monoid case', Proc. Edinburgh Math. Soc. 42 (1999), 497-520.

[20] - 'E*-unitary inverse semigroups', in: Semigroups, Algorithms, Automata and Languages (eds. G. M. S. Gomes, J.-E. Pin and P. Silva) (World Scientific, Singapore, 2002), pp. 155-194.

[21] D. B. McAlister, 'One-to-one partial right translations of a right cancellative semigroup', J. Algebra 43 (1976), 231-251.

[22] - 'A random ramble in inverse semigroups II: an introduction to $E^{*}$-unitary inverse semigroups-from an old fashioned perspective', in: Semigroups and Languages, Lisbon 2002 (eds. I. M. Araújo, M. J. J. Branco, V. H. Fernandes and G. M. S. Gomes) (World Scientific, Singapore, 2004), pp. 133-150.

[23] D. B. McAlister and R. McFadden, 'Zig-zag representations and inverse semigroups', J. Algebra 32 (1974), 178-206.

[24] S. W. Margolis, 'Embedding semigroups in groups', Talk at University of York Algebra Seminar, 21 May 2003.

[25] J. Meakin and M. Sapir, 'Congruences on free monoids and submonoids of polycyclic monoids', J. Aust. Math. Soc. Ser. A 54 (1993), 236-253.

[26] M. Nivat and J.-F. Perrot, 'Une généralisation du monoïde bicyclique', C. R. Acad. Sci. Paris Sér. A-B 271 (1970), A824-A827.

[27] L. Paris, 'Artin monoids inject in their groups', Comment. Math. Helv. 77 (2002), 609-637.

[28] D. Rees, 'On the group of a set of partial transformations', J. London Math. Soc. 22 (1948), 281-284.

[29] B. Steinberg, 'The uniform word problem for groups and finite Rees quotients of $E$-unitary inverse semigroups', J. Algebra 266 (2003), 1-13.

[30] M. B. Szendrei, 'A generalization of McAlister's $P$-theorem for $E$-unitary regular semigroups', Acta Sci. Math. (Szeged) 51 (1987), 229-249.

[31] A. Veloso da Costa, 'Graph products of monoids', Semigroup Forum 63 (2001), 247-277.

[32] _ ' 'On graph products of automatic monoids', Theor. Inform. Appl. 35 (2001), 403-417.

JOHN FOUNTAIN, Department of Mathematics, University of York, Heslington, York YO10 5DD, UK

e-mail: jbf1@york.ac.uk

MARK KAMBITES, School of Mathematics, University of Manchester, Manchester M13 9PL, UK

e-mail: Mark.Kambites@manchester.ac.uk 